\title{
False and true pre-treatment predictors of weight loss in obese patients starting a program for lifestyle change
}

\author{
Barbara Cresci $\cdot$ Laura Pala $\cdot$ Roberta Poggiali • \\ Cosetta Guarnieri · Edoardo Mannucci • \\ Michela Bigiarini · Carlo Maria Rotella
}

Received: 16 January 2014/ Accepted: 10 April 2014/Published online: 11 May 2014

(C) The Author(s) 2014. This article is published with open access at Springerlink.com

\begin{abstract}
Purpose Weight loss treatment effectiveness and costeffectiveness may be improved by the identification of patients who are more prone to participate and gain benefit from specific interventions. Aim of the present study is to identify easily available additional predictors of weight loss among data usually present in the medical records of obese/overweight patients attending an outpatient clinic for a non-pharmacological lifestyle change program.

Results 268 patients, 74 men and 195 women (age $43.2 \pm 11.9$ years, BMI $38.9 \pm 6.8 \mathrm{~kg} / \mathrm{m}^{2}$ ) were enrolled. Among these patients, only $35.6 \%$ men and $22.7 \%$ women completed the 6-month protocol. Among participants, $50.7 \%$ lost at least $5 \%$ initial body weight after 6 months (SUCCESSES), while $49.3 \%$ failed (FAILURES). Baseline nutritional parameters (total Kcal, lipid, carbohydrate, protein and alcohol intake) were not significantly different in successes when compared to failures, while a significant difference between groups was observed for baseline diastolic blood pressure (DBP); free fat mass (FFM); muscle mass (MM); total body water (TBW); HDL cholesterol; ALT; AST; $\gamma$ GT. After dividing into quartiles
\end{abstract}

B. Cresci · L. Pala

Endocrinology Unit, Careggi University Hospital, Florence, Italy

R. Poggiali · C. Guarnieri

Dietetic Unit, Careggi University Hospital, Florence, Italy

E. Mannucci

Diabetes Agency, Careggi University Hospital, Florence, Italy

M. Bigiarini · C. M. Rotella $(\bowtie)$

Department of Biomedical Experimental and Clinical Sciences and Obesity Agency, University of Florence, Viale Pieraccini 6, 50134 Florence, Italy

e-mail: carlomaria.rotella@unifi.it the not-normally distributed variables, successes had AST values above median ( $3 \mathrm{rd}$ and 4 th quartiles; $\chi^{2}=0.003$ ). At multivariate analysis (linear regression), the OR was $3.34(1.42-7.85 ; p=0.006)$.

Conclusions In our patients, baseline liver enzyme levels (AST in particular), but not baseline quantitative and qualitative dietary intake, were significantly different in successes versus failures and could therefore represent a predictor of success. In conclusion, AST could represent a usually available biomarker that could be used as a predictor of outcome (weight loss) in obese patients starting a lifestyle change program.

Keywords Obesity - Outcome $\cdot$ Muscle mass $\cdot$ Weight loss

\section{Introduction}

Weight loss programs for obese patients, particularly those lifestyle change-oriented and without any pharmacological treatment, are known to be time consuming and usually show low percentages of success in terms of weight loss for a relevant number of different reasons [1]. At this purpose, weight loss treatment effectiveness and cost-effectiveness may be improved, not only by the identification of factors affecting weight loss, but mainly by the identification of patients who are sufficiently motivated and thus more able to participate and gain benefit from the intervention.

Many parameters have been identified with a potential capacity of predicting success/failure in a weight loss program [2-4]. Among these, the most suggestive could be considered: self-motivation [5-7]; self-efficacy [8]; active lifestyle [9]; more relevant initial weight loss [10]; social and/or family support $[11,12]$. 
Our group has already explored this area, reporting that therapeutic success is predicted by basal TRE-MORE test scores and by estimated muscle mass. In fact, we have found that a higher muscle mass, as estimated through bioimpedance analysis (BIA), is associated with a greater weight loss in obese patients [13]. Moreover, we demonstrated that therapeutic success is predicted by TRE-MORE test scores. In particular, TRE-MORE total score is a predictor of failure, but not of attendance, whereas drop-out patients showed a lower score only in TREMORE-3 subscale which investigates lifestyle habits [14, 15].

A substantial body of literature exists suggesting that weight loss can be achieved by varying the macronutrient distribution and composition of dietary factors. Champagne et al. [16] have investigated the effect of dietary intake modifications on weight loss and maintenance during an intensive behavioral weight loss program, concluding that success was mainly associated with increased intake of proteins, fruits and vegetables, and low-fat dairy. Moreover, many studies have explored the possibility of using the variation in the intake of different macronutrients as predictors of weight loss [17-19]. Very few studies, on the contrary, explore pre-treatment dietary habits impact on treatment success. Recently, Byrne et al. [20] evaluated the effects of pre-treatment self-efficacy for diet and exercise, as well as changes in self-efficacy occurring during treatment, on weight loss success, demonstrating that treatment attendance and changes in exercise self-efficacy during treatment were stronger predictors of weight loss than changes in diet.

It is well known that obesity is frequently associated to many other morbidities. The majority of these complications are related to comorbid conditions that include coronary artery disease, hypertension, type 2 diabetes mellitus, respiratory disorders and dyslipidemia. Therefore, patients starting a weight loss program are usually screened for biohumoral parameters related to these complications, such as lipid profile, glycemia, insulinemia, blood pressure levels and liver function, the potentiality of which as predictors has not yet been demonstrated.

Aim of the present study is to identify easily available predictors that could be used as additional predictors of weight loss among data present in the medical records of obese/overweight patients attending an outpatient clinic.

\section{Methods}

\section{Patients}

The study was carried out in the Outpatient Clinic of the Obesity Agency of the University of Florence. Complete baseline data including blood samples results were
Table 1 Inclusion and exclusion criteria of patients

Inclusion criteria (A)
Obesity or overweight (BMI $\geq 27 \mathrm{~kg} / \mathrm{m}^{2}$ )
Age $18-65$
Residence within $40 \mathrm{~km}$ from the Clinic
Informed consent
Exclusion criteria (B)
Patients living at distances more than $40 \mathrm{~km}$
Uncontrolled endocrine disorders, such as hypo- or
hyperthyroidism
Diabetes
Pregnancy
Illiteracy, or inadequate knowledge of the Italian language
Any condition interfering with the possibility of regular physical
exercise (e.g., severe cardiac dysfunction, severe respiratory
insufficiency, major neurologic disorders, etc.)
Diagnosis of major depression, bipolar disorder, obsessive-
compulsive disorder, schizophrenia, or mental retardation
Current treatment with antipsychotics, antiepileptics, tricyclic
antidepressants, or lithium
Intention to move more than 40 km far from Florence in the
following 12 months

collected for 268 patients out of the enrolled 331 seeking treatment for overweight/obesity in our outpatient clinic [15].

Inclusion and exclusion criteria are shown in Table 1.

Before the collection of data, during the first routine visit, the procedures of the study were fully explained; after that, the patients were asked to provide their written informed consent to the participation to the study. The study protocol had been previously approved by the Local Ethical Committee. The treatment protocol was carried out as previously described [15].

\section{Measurements}

At baseline, an Endocrinologist collected an accurate medical history, performed a complete physical examination and measured anthropometric parameters (BMI, waist circumference, blood pressure and heart rate). Body fat (BF) and lean body mass were estimated through BIA, performed by a tetrapolar single frequency $(50 \mathrm{kHz})$ phasesensitive impedance analyzer (QUANTUM/S; AkernSrl, Firenze, Italy). In the same day, the patients also met a dietician (R.P. and C.G.) who assessed usual food intake on the basis of a 30-day recall, using a dedicated software (Gedip by Solutions S.n.c, Italy), together with photo atlas for the determination of portions (Scotti-Bassani Institute, Italy). A goal of a $500 \mathrm{kcal} /$ day reduction from usual food intake was agreed upon with the patient. To reach that objective, the patients were asked to self-monitor their food intake for at least a week before each monthly follow-up 
visit with the dietitian [15]. Blood samples were drawn after 12-h overnight fast for glucose (Beckman instruments, Fullerton, CA, USA), TSH (Electrochemiluminescent, Modular Roche, Milan, Italy), transaminases, gammaGT, insulin (electrochemiluminescence immunoassay, Roche Diagnostics, Mannheim, Germany), cholesterol, HDL-cholesterol, triglycerides (for lipid panel: Abbott Bichromatic Analyser (Abbott Diagnostics, South Pasadena, CA, USA). All laboratory determinations were performed in the central Laboratory of Careggi Hospital in Florence.

Final measures, including BMI and waist, were considered those collected after 6 months from the enrollment in the protocol.

\section{Definition of outcomes}

Therapeutic success was defined as a weight loss at 6 months of at least $5 \%$ from baseline to assess this outcome.

\section{Statistical analysis}

Continuous variables were reported as mean \pm standard deviations (SD), whereas categorical variables were reported as median and limits of confidence. Betweengroup comparisons of continuous variables were performed using unpaired Student's $t$ test or Mann-Whitney $U$ test, for variables with normal or non-normal distribution, respectively. Correlation analyses were performed with Spearman's method. Logistic regression analyses were applied for dichotomous outcomes (successes vs. failures).

All analyses were performed using SPSS for Windows 15.0 (Chicago Inc, USA).

\section{Results}

268 patients, 74 men and 195 women (age $43.2 \pm 11.9$ years, weight $105.6 \pm 22.6 \mathrm{~kg}$, BMI $38.9 \pm 6.8 \mathrm{~kg} / \mathrm{m}^{2}$, waist $116.1 \pm 16.2 \mathrm{~cm}$ ) were enrolled. Among these patients, only $26(35.6 \%)$ men and $44(22.7 \%)$ women completed the 6-month protocol. Among participants, $50.7 \%(n=70)$ lost at least $5 \%$ initial body weight after 6 months (and will be thereafter called SUCCESSES), while $49.3 \%(n=68)$ failed (FAILURES).

Baseline nutritional parameters, assessed as previously described, were also analyzed (Table 2). In particular, total Kcal intake, lipid intake, carbohydrate intake, protein intake, together with baseline alcohol intake were not significantly different in successes when compared to failures. Furthermore, the spontaneous difference in total kcal intake, lipid intake, carbohydrate intake, protein
Table 2 Baseline nutritional parameters: differences between successes and failures

\begin{tabular}{llll}
\hline Baseline intake & Successes & Failures & $p$ \\
\hline Kcal & $2,265.1 \pm 887.4$ & $2,032.7 \pm 856.1$ & 0.121 \\
Proteins (g) & $88.3 \pm 33.8$ & $83.7 \pm 31.5$ & 0.513 \\
Lipids (g) & $82.1(68.3-106.1)$ & $81.0(65.0-102)$ & 0.784 \\
Saturated lipids (g) & $25.0 \pm 10.4$ & $22.2 \pm 12.1$ & 0.228 \\
Carbohydrates (g) & $293.8 \pm 136.6$ & $253.4 \pm 118.2$ & 0.067 \\
Alcohol (g) & $5.0(1.2-23.5)$ & $4.4(1.0-19.9)$ & 0.511 \\
\hline
\end{tabular}

Data are shown as media $\pm \mathrm{SD}$, whereas categorical variables were reported as median and limits of confidence

Table 3 Differences between baseline data of successes (losing at least $5 \%$ of initial body weight at the intention-to-treat analysis) and failures (completers losing $<5 \%$ of initial body weight and dropouts)

\begin{tabular}{lccl}
\hline & Successes & Failures & $p$ \\
\hline DBP (mmHg) & $78 \pm 6$ & $75 \pm 9$ & 0.033 \\
FFM (\%) & $62.8 \pm 15.1$ & $57.8 \pm 9.3$ & 0.021 \\
MM (\%) & $45.2 \pm 12.6$ & $40.6 \pm 7.6$ & 0.011 \\
TBW (\%) & $46.3 \pm 11.1$ & $42.5 \pm 6.7 \%$ & 0.021 \\
HDL (mg/dl) & $44.9 \pm 11.9$ & $50.5 \pm 14.8$ & 0.020 \\
ALT (mg/dl) & $29.6 \pm 30.8$ & $20 \pm 5$ & 0.017 \\
AST (mg/dl) & $40.4 \pm 51.2$ & $25.5 \pm 12.3$ & 0.027 \\
$\gamma$ GT (mg/dl) & $38.6 \pm 52.3$ & $22.2 \pm 11.4$ & 0.019 \\
\hline
\end{tabular}

Data are shown as media \pm SD

intake, alcohol intake recorded after 1-week self-monitoring was not significantly different in successes when compared to failures.

Differences between baseline data of successes (losing at least $5 \%$ of initial body weight at the intention-to-treat analysis) and failures (completers losing $<5 \%$ of initial body weight and drop-outs) are summarized in Table 3. A significant difference was observed only for diastolic blood pressure (DBP) (78 \pm 6 vs. $75 \pm 9 \mathrm{mmHg} ; p=0.033)$; free fat mass (FFM) $(62.8 \pm 15.1$ vs. $57.8 \pm 9.3 \%$; $p=0.021)$; muscle mass $(\mathrm{MM})(45.2 \pm 12.6$ vs. $40.6 \pm$ $7.6 \% ; p=0.011)$; total body water (TBW) $(46.3 \pm 11.1$ vs. $42.5 \pm 6.7 \% ; p=0.016)$; HDL $(44.9 \pm 11.9$ vs. $50.5 \pm 14.8 \mathrm{mg} / \mathrm{dl} ; \quad p=0.020) ;$ ALT $(29.6 \pm 30.8$ vs. $20 \pm 5 \mathrm{mg} / \mathrm{dl} ; p=0.017$ ); AST ( $40.4 \pm 51.2$ vs. $25.5 \pm 12.3$ $\mathrm{mg} / \mathrm{dl} ; p=0.027) ; \gamma \mathrm{GT}(38.6 \pm 52.3$ vs. $22.2 \pm 11.4 \mathrm{mg} / \mathrm{dl}$; $p=0.019$ ).

None of these differences was retained at multivariate analysis with only one exception. In fact, after dividing into quartiles the not-normally distributed variables, it resulted that successes have AST values above median (3rd and 4th quartiles; $\left.\chi^{2}=0.003\right)$, even if a trend was observed for ALT (3rd and 4th quartiles; $\chi^{2}=0.068$ ) and $\gamma$ GT (3rd and 4 th quartiles; $\left.\chi^{2}=0.074\right)$. At multivariate analysis 
(logistic regression), after adjusting for age and waist, the OR for AST was $3.34(1.42-7.85 ; p=0.006)$.

\section{Discussion}

The present study tries to identify new possible predictors of outcome in a non-pharmacologic lifestyle change-centered weight loss program. For this purpose, we analyzed a series of baseline blood parameters, which are usually studied in obese patients to assess the presence of eventual comorbidities. In particular, successes resulted to have GOT/AST values above median.

It is well known that obesity is frequently associated with a cluster of risk factors including dysglycemia, hypertension and dyslipidemia, in a few words defined by the concept of metabolic syndrome (MS). As a consequence, obese patients are often affected by non-alcoholic fatty liver disease (NAFLD). Impaired hepatic fatty acid (FA) turnover together with insulin resistance are key players in NAFLD pathogenesis [21]. Most individuals are asymptomatic and are usually discovered incidentally because of abnormal liver function tests. Elevated liver biochemistry is found in $50 \%$ of patients with simple steatosis. Change in liver function tests is considered as surrogate marker of liver injury and non-alcholic fatty liver disease (NAFLD). Previous studies have demonstrated that circulating concentration of liver function tests like $\gamma$ glutamyltransferase $(\gamma \mathrm{GT})$, alanine aminotransferase (ALT) and aspartate aminotransferase (AST) is increased in individuals with insulin resistance and the metabolic syndrome [22]. In addition, these components of liver function tests have been shown to be positively associated with the risk of future type 2 diabetes [23, 24]. In particular, available data indicate moderate associations of ALT and $\gamma \mathrm{GT}$ with risk of type 2 diabetes events, and no evidence for an increased risk with AST [25, 26].

Moreover, recent data [27] speculate that abnormal levels of ALT and AST are associated with a deregulation of normal amino acid metabolism in the liver, including aromatic amino acid, and then special compounds such as glutamate are released into the general circulation. This hypothesis attempts to illustrate the critical role of the "liver metabolism" in the pathogenesis of the MS and postulates that before the liver becomes fatty, abnormal levels of liver enzymes might reflect high levels of hepatic transamination of amino acids in the organ. Anyway, elevated transaminase levels could represent an early at-risk situation of pre-NAFLD.

In our patients, baseline liver enzyme levels (AST in particular), but not baseline quantitative (total Kcal) and qualitative (lipids, carbohydrates, proteins, alcohol) dietary intake, were significantly different in successes versus failures and could therefore represent a predictor of success. Successes have AST values above median. A possible explanation could be that AST is raised in acute liver damage, but is also present in red blood cells, and cardiac and skeletal muscle and is therefore not specific to the liver. For instance, in athletes, the interpretation of serum aminotransferases concentrations should consider the release of AST from muscle and of ALT mainly from the liver [28]. Therefore, we could speculate that the predictive value of AST but not of ALT or $\gamma \mathrm{GT}$ in our population could be related to the significantly different amount of baseline muscle mass in the two populations. This could be consistent with our previous findings that successes have better baseline grade of fitness in terms of initial muscle mass when compared to failures [15].

Both genetic and environmental factors have been proposed to be involved in the etiology of NAFLD. Thus, nutrition is reasonably considered to be a potential environmental factor affecting the risk for this disease. Although there is consistent evidence that overweight due to energy overconsumption increases the risk for and the prevalence of fatty liver, the role of diet composition, in terms of macro- or micronutrients, in the pathogenesis of the disease remains controversial [29]. Dietary habits shown by patients before starting the lifestyle change program have not been demonstrated to be significantly different between those losing at least $5 \%$ of initial body weight and failures. The same result was obtained comparing the basal and 1 week after quantitative and qualitative dietary intake, spontaneously modified by patients without the dietician intervention. Anyway, we should point out that both baseline total Kcal and carbohydrate intake, even if not reaching the statistical significance, show different values when comparing successes and failures. In particular, failures report lower total Kcal intake and lower carbohydrate intake before entering the program. It should be taken into consideration that data regarding qualitative and quantitative dietary intake were collected based on information self-reported by patients. The tools used by the operators, such as a dedicated software and a photo atlas representing different portion sizes, were aimed at reducing the bias, but the problem related to an altered perception from the patient's point of view could nevertheless be still present.

Moreover, despite worldwide guidelines and recommendations, research examining diet composition for the management of obesity remains controversial. Previous studies have found that low-fat diets promote short-term weight loss [30]; however, some studies suggest that lowcarbohydrate, high-protein, and high-fat diets may also result in substantial weight loss [31]. We can therefore conclude that a substantial body of literature exists suggesting that weight loss can be achieved by varying the 
macronutrient distribution and composition of dietary factors, even if evidence for type of diet on long-term weight maintenance remains debatable $[16,32]$. On the other hand, few data are present regarding quality of eating patterns as predictors of weight loss. Hart et al. [33] demonstrated that early changes in eating habits, but not baseline eating behaviors may promote greater BMI reductions in a cohort of adolescent patients. These findings are consistent with our data, even if referred to a completely different population.

Based on our data, we can at the moment conclude that neither usual dietary habits nor self-management operated by patients based on personal beliefs could represent a good predictor of success for this kind of programs. Finally, these results confirm once again that baseline grade of fitness (i.e., initial muscle mass) is a better predictor of success, rather than dietary habits, when starting a lifestyle modification program, as already demonstrated by our group [15].

Moreover, we can conclude that AST could represent a usually available biomarker that could be used as a predictor of weight loss in obese patients starting a lifestyle change program. Nevertheless, this should be considered as a pilot study which includes a reasonable number of patients and therefore the efficacy of present data, which aim to identify a weight loss predictor of outcome, will need to be verified through specifically designed longerterm randomized clinical trials enrolling a larger number of patients.

Acknowledgments Present study was supported by grants to CMR by the Italian Ministry of Health (Rome, Italy), by the Regione Toscana Department of Health (Firenze, Italy), by Ely Lilly Italy and by Fondazione Cassa di Risparmio di Firenze, Italy. For this study CMR received unconditioned grants from Ely Lilly Italy and from Fondazione Cassa di Risparmio di Firenze, Italy.

Conflict of interest For this study the authors declare no conflict of interest.

Open Access This article is distributed under the terms of the Creative Commons Attribution License which permits any use, distribution, and reproduction in any medium, provided the original author(s) and the source are credited.

\section{References}

1. Greaves CJ, Sheppard KE, Abraham C, Hardeman W, Roden M, Evans PH, Schwarz P, IMAGE Study Group (2011) Systematic review of reviews of intervention components associated with increased effectiveness in dietary and physical activity interventions. BMC Public Health 11:119-130. doi:10.1186/1471-245811-119-130

2. Rieder S, Ruderman A (2007) The development and validation of the weight management support inventory. Metabolism 8:39-47

3. Teixeira PJ, Going SB, Houtkooper LB et al (2004) Pre-treatment predictors of attrition and success weight management in women. Int J Obes Relat Metab Disord 28:1124-1133
4. Teixeira PJ, Going SB, Sardinha LB et al (2005) A review of psychosocial pre-treatment predictors of weight control. Obes Rev 6(1):43-65

5. Cresci B, Rotella CM (2009) Motivational readiness to change in lifestyle modification programs. Eat Weight Disord 14:e158 e162

6. Teixeira PJ, Silva MN, Countinho SR et al (2009) Mediators of weight loss and weight loss maintenance in middle-age women. Obesity (Silver Spring) 18(4):725-735. doi:10.1038/oby.2009. 281

7. Teixeira PJ, Palmeira AL, Branco TL et al (2004) Who will lose weight? A re-examination of predictors of weight loss in women. Int J Behav Nutr Phys Act. 1(1):12

8. Edell BH, Edington S, Herd B, O'Brain RM, Witkin G (1987) Self-efficacy and self-motivation as predictors of weight loss. Addict Behav 12:63-66

9. Annesi JJ (2011) Behaviorally supported exercise predicts weight loss in obese adults through improvements in mood, self-efficacy, and self-regulation, rather than by caloric expenditure. Perm $\mathbf{J}$ 15(1):23-27

10. Elfhag K, Rössner S (2010) Initial weight loss is the best predictor for success in obesity treatment and sociodemographic liabilities increase risk for drop-out. Patient Educ Couns 79(3):361-366

11. Kiernan M, Moore SD, Schoffman DE et al (2012) Social support for healthy behaviors: scale psychometrics and prediction of weight loss among women in a behavioral program. Obesity (silver Spring) 20(4):756-764. doi:10.1038/oby.2011.293

12. Pinelli NR, Brown MB, Herman WH, Jaber LA (2011) Family support is associated with success achieving weight loss in a group lifestyle intervention for diabetes prevention in Arab Americans. Ethn Dis 21(4):480-484

13. Dicembrini I, Pala L, Cresci B, Cremasco F, Rotella CM (2010) Predictors of weight loss in the clinical management of obese patients: the relevance of body composition. Obes Metab 6:29-33

14. Cresci B, Castellini G, Pala L, Rotella CM, Ricca V (2011) Motivational readiness for treatment in weight control programs: the TREatment Motivation REadiness (TRE-MORE) test. J Endocrinol Invest 34:e70-e77

15. Cresci B, Castellini G, Pala L, Bigiarini M, Romoli E, Poggiali R, Guarnieri C, Biffi B, La Ferlita T, Ricca V, Mannucci E, Rotella CM (2013) Fit and motivated: outcome predictors in patients starting a program for lifestyle change. Obes Facts 6(3):279-287. doi:10.1159/000353433

16. Champagne CM, Broyles ST, Morand LD (2011) Dietary intakes associated with successful weight loss and maintenance during the weight loss maintenance trial. J Am Diet Assoc 111(12):1826-1835

17. Canfi A, Gepner Y, Schwarzfuchs D, Golan R et al (2011) Effect of changes in the intake of weight of specific food groups on successful body weight loss during a multi-dietary strategy intervention trial. J Am Coll Nutr 30(6):491-501

18. Hart CN, Jelalian E, Raynor HA, Mehlenbeck R (2010) Early patterns of food intake in an adolescent weight loss trial as predictors of BMI change. Eat Behav 11(4):217-222

19. Barone Gibbs B, Kinzel LS, Pettee Gabriel K et al (2012) Shortand long-term eating habit modification predicts weight change in overweight, postmenopausal women: result from the WOMAN study. J Acad Nutr Diet 112(9):1347-1355. doi:10.1016/j.jand. 2012.06.012

20. Byrne S, Barry D, Petry NM (2012) Predictors of weight loss success. Exercise vs. dietary self-efficacy and treatment attendance. Appetite 58:695-698

21. Levene AP, Goldin RD (2012) The epidemiology, pathogenesis and histopathology of fatty liver disease. Histopathology 61:141-152 
22. Hanley AJ, Williams K, Festa A, Wagenknecht LE, D'Agostino RB Jr et al (2004) Elevations in markers of liver injury and risk of type 2 diabetes: the insulin resistance atherosclerosis study. Diabetes 53:2623-2632

23. Fraser A, Harris R, Sattar N, Ebrahim S, Davey Smith G et al (2009) Alanine aminotransferase, gamma-glutamyltransferase, and incident diabetes: the British Women's Heart and Health Study and meta-analysis. Diabetes Care 32:741-750

24. Abbasi A, Bakker SJ, Corpeleijn E, van der ADL, Gansevoort RT, Gans RO, Peelen LM, van der Schouw YT, Stolk RP, Navis G, Spijkerman AM, Beulens JW (2012) Liver function tests and risk prediction of incident type 2 diabetes: evaluation in two independent cohorts. PLoS One 7(12):e51496. doi:10.1371/journal.pone.0051496

25. Kunutsor SK, Apekey TA, Walley J (2013) Liver aminotransferases and risk of incident type 2 diabetes: a systematic review and meta-analysis. Am J Epidemiol 178(2):159-171. doi:10. 1093/aje/kws469

26. Sattar N (2012) Biomarkers for diabetes prediction, pathogenesis or pharmacotherapy guidance? Past, present and future possibilities. Diabet Med 29:5-13

27. Sookoian Silvia, Pirola CJ (2012) Alanine and aspartate aminotransferase and glutamine-cycling pathway: their roles in pathogenesis of metabolic syndrome. World J Gastroenterol 18(29): 3775-3781
28. Banfi G, Colombini A, Lombardi G, Lubkowska A (2012) Metabolic markers in sports medicine. Adv Clin Chem 56:1-54

29. Mouzaki M, Allard JP (2012) The role of nutrients in the development, progression, and treatment of nonalcoholic fatty liver disease. J Clin Gastroenterol 46:457-467

30. Astrup A, Grunwald GK, Melanson EL, Saris WH, Hill JO (2000) The role of low-fat diets in body weight control: a meta-analysis of ad libitum dietary intervention studies. Int J Obes Relat Metab Disord 24:1545-1552

31. Gardner CD, Kiazand A, Alhassan S, Kim S, Stafford RS, Balise RR, Kraemer HC, King AC (2007) Comparison of the Atkins, Zone, Ornish, and LEARN diets for change in weight and related risk factors among overweight premenopausal women: the A TO Z weight loss study: a randomized trial. JAMA 297: 969-977

32. Canfi A, Gepner Y, Schwarzfuchs D, Golan R et al (2011) Effect of changes in the intake of weight of specific food groups on successful body weight loss during a multi-dietary strategy intervention trial. J Am Coll Nutr 30(6):491-501

33. Hart CN, Jelalian E, Raynor HA, Mehlenbeck R, Lloyd-Richardson EE, Kaplan J, Flynn-O'Brien K, Wing RR (2010) Early patterns of food intake in an adolescent weight loss trial as predictors of BMI change. Eat Behav 11(4):217-222. doi:10.1016/j. eatbeh.2010.05.001 